\title{
Penser en interdisciplinarité dans une UMR de sciences humaines et sociales (2007-2011)
}

\section{Daniel Russo}

\section{(2) OpenEdition \\ 1 Journals}

\section{Édition électronique}

URL : https://journals.openedition.org/cem/12112

DOI : $10.4000 /$ cem. 12112

ISSN : 1954-3093

Éditeur

Centre d'études médiévales Saint-Germain d'Auxerre

\section{Édition imprimée}

Pagination : 305-318

ISSN : $1623-5770$

\section{Référence électronique}

Daniel Russo, «Penser en interdisciplinarité dans une UMR de sciences humaines et sociales (2007-2011) », Bulletin du centre d'études médiévales d'Auxerre | BUCEMA [En ligne], 15 | 2011, mis en ligne le 20 septembre 2011, consulté le 22 septembre 2022. URL : http://journals.openedition.org/ cem/12112; DOI : https://doi.org/10.4000/cem.12112

Ce document a été généré automatiquement le 22 septembre 2022.

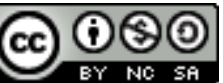

Creative Commons - Attribution - Pas d'Utilisation Commerciale - Partage dans les Mêmes Conditions 4.0 International - CC BY-NC-SA 4.0

https://creativecommons.org/licenses/by-nc-sa/4.0/ 


\title{
Penser en interdisciplinarité dans une UMR de sciences humaines et sociales (2007-2011)
}

\author{
Daniel Russo
}

1 Ces réflexions me sont inspirées par l'expérience que j'ai acquise au cours des quatre années (janvier 2007-janvier 2011) que j'ai effectuées comme son directeur à la tête de l'Unité mixte de recherches (UMR) 5594 Artehis - Archéologie, terre, histoire, société (l'acronyme renvoyant aussi à histoire de l'art, depuis la préhistoire jusqu'au Moyen Âge), ainsi que par les enseignements que j'ai retirés du séminaire interdisciplinaire que j'y ai conduit sur la problématique « nature/culture ${ }^{1}$.

2 Je ne reviendrai pas ici sur les questions que je pointais dans le bilan de l'UMR, ni davantage sur celles que j'abordais dans le projet de travail élaboré dans le cadre de la nouvelle direction: qu'il me suffise d'y renvoyer le lecteur pour plus d'informations, tant sur les orientations générales que j'essayais d'impulser que sur les axes de recherches que j'y proposais ${ }^{2}$. Je préciserai, en revanche, à partir de ma formation d'historien et de mon expérience d'historien de l'art, soucieux de travailler dans le champ des sciences humaines et sociales, ouvert à celui des sciences de la vie et de la terre, les conditions dans lesquelles je fus amené à repenser le binôme "nature/ culture » et les enjeux de cette réflexion que je voulus, d'emblée, à la rencontre des disciplines qui composaient le laboratoire ${ }^{3}$. À mon intérêt trois raisons s'imposaient avant tout, que je vais essayer de résumer :

- la nécessité de « penser la pensée » à l'œuvre dans les différents champs de la recherche menée en archéologie, en histoire, en histoire de l'art, pour m'en tenir aux disciplines académiques reconnues et regroupées au sein de l'UMR, puis d'en esquisser, pour ainsi dire, une « ethnographie » pour les années 2006-2011 en interrogeant la notion même de « culture ${ }^{4}$;

- l'opportunité de situer ces interrogations au cœur même du problème de l'unité et de la diversité de l'humain et, plus largement encore, du vivant, afin de reprendre, en le poursuivant, un mouvement global bien lancé dans les sciences humaines et sociales au 
moins depuis les années 1920-1930, et notamment en anthropologie : ceci tant aux différents niveaux du processus psychologique, donc interne à la pensée elle-même, qu'à ceux du processus culturel considérant la pensée comme « un fait social » et l'idée comme « une chose sociale ${ }^{5}$;

- la possibilité de «figurer autrement » les contours de la recherche dans la vie de laboratoire, donc de redéfinir ses liens avec la société qui l'entoure aux plans régional, national et international ${ }^{6}$. Ce sont les raisons qui formèrent la trame du projet que je conçus et que je m'efforçai d'articuler, pour le meilleur, aux pratiques et aux méthodes en cours.

\section{L'art comme expérience du social}

3 Je les expliquerai à présent dans le temps de mon écriture et, ainsi mis à distance par le temps qui est passé, en recourant de manière trop commode, peut-être, à trois expressions qui disent, chacune différemment, assez bien toutefois, l'intention qui fut la mienne, à partir de l'histoire de l'art comprise comme une histoire des choses et de leurs pratiques dans le monde: la perception historicisée des systèmes culturels du vivant, au premier chef desquels je placerai le système culturel de l'art, dont je me suis servi comme base de départ; la refiguration de la pensée sur le social qui en découle; l'analogie efficace avec le textuel ${ }^{7}$, sans cesse au travail dans ma réflexion, et à la base d'une nouvelle politique de laboratoire fondée sur les sources et sur les ressources pour étudier le document et le monument.

4 Les concepts que je fus conduit à penser, puis à définir, provenaient de mon travail même de recherche en histoire et en histoire de l'art du Moyen Âge et, entre autres lectures, des points de jonction possible avec les autres sciences humaines et sociales, aux premiers rangs desquelles l'anthropologie et la philosophie. Étaient sous-jacentes les questions de validité du discours scientifique et, plus que toute autre préoccupation, celles relatives au statut de la preuve: c'est par ce biais que je cherchais aussi à analyser et à comprendre les conditions de validation des résultats dans le champ des sciences, à partir de l'étude des mots et de leur mise en ordre textuel, à partir aussi de la conceptualisation du langage et de ses effets, par Ludwig Wittgenstein (1889-1951) ${ }^{8}$. De la même manière, les travaux des philosophes des sciences sur les images produites dans les laboratoires et au cours des phases capitales du processus de leurs recherches, ont élargi mes perspectives d'enquête en m'éloignant définitivement de la référence au " réel ${ }^{9}$ " et en approfondissant les relations improbables entre "religion, art et science ${ }^{10}:$ je retiendrai, entre autres apports, la notion problématique d'objectivité des choses dans le monde et celle de flux des images ${ }^{11}$. Dans tous les cas, la confrontation de ces points de vue avec mes propres intérêts s'est révélée d'une très grande portée heuristique.

5 C'est dans ces conditions que je reformulai une interrogation naguère posée par Michael Baxandall (1933-2008) en pensant à l'histoire du discours de la critique d'art, et en l'étendant à tout le «champ » des objets culturels et à l'étude qu'on en fait : où est encore l'art, où est encore l'histoire ${ }^{12}$ ? Et quelle est la part qui leur revient dans la description, puis la compréhension d'un système culturel? Dans des ouvrages fondamentaux parus en 1971, en 1972 et en 1980, respectivement autour de la formation d'un discours critique sur l'art à l'époque de Giotto et des premiers cercles humanistes, ensuite sur la peinture comme l'expérience sociale la plus réussie, et la mieux partagée, par la haute société $d u \mathrm{Xv}^{\mathrm{e}}$ siècle en Italie, enfin sur les sculpteurs sur 
bois de tilleul dans l'Allemagne à la charnière des $\mathrm{xv}^{\mathrm{e}}$ et $\mathrm{xvI}^{\mathrm{e}}$ siècles ${ }^{13}$, Michael Baxandall a circonscrit les différents domaines de l'expérience sociale des arts visuels pour des périodes précises et dans des milieux bien repérés dans les textes qu'ils produisirent: lettres et traités des humanistes du nord de la péninsule italienne, entre 1350 et 1450 ; chroniques d'histoire urbaine, lettres, traités d'humanistes ou sermons de religieux mendiants tout au long $\mathrm{du} \mathrm{xv}^{\mathrm{e}}$ siècle, toujours en Italie ; contrats urbains et termes techniques du marché pour les artisans du bois et leur production de sculptures dans les régions méridionales de l'Allemagne, entre Moyen Âge et modernité. C'est, du reste, à ces niveaux de compréhension socioculturelle qu'il fut lu par Clifford Geertz et Pierre Bourdieu, notamment ${ }^{14}$ : l'un discutant son apport à l'étude de la culture plus vaste $d u x^{e}$ siècle italien, en s'interrogeant sur les relations entre l'art et le social, qu'il qualifie d'interactives et de complémentaires; l'autre présentant à partir de ses observations sur les peintures du Quattrocento un « tableau » sur la sociologie du voir, et du faire voir, à l'époque ${ }^{15}$. À la lecture de ces livres, parmi les plus stimulants de la décennie 1970-1980, je devais surtout retenir le concept de l'art comme celui d'une "expérience sociale partagée " ${ }^{16}$ et en déduire quelques lignes directrices de travail qui guidèrent mon analyse et la recentrèrent dans le champ des sciences humaines et sociales. Je distinguais ainsi quatre principes qui servirent de fondements à mes orientations de recherches et à la définition des axes de travail du laboratoire.

\section{Principes et axes de travail en sciences humaines et sociales}

6 D'abord, et avant tout, l'attention à l'objet, ou plutôt à la « chose » étudiée sous ses différents aspects et dans ses multiples composants, toujours en situation, c'est-à-dire en tenant le plus grand compte de son inscription dans le texte d'une culture donnée ce texte étant produit par l'unité dans la diversité que forment ses contextes, ses paratextes, mais aussi ses hors-textes -, à l'intérieur de la configuration des sources et des ressources documentaires, que je me proposais d'intituler à la suite des linguistes un corpus orienté autant vers les possibilités de l'inventaire, voire du catalogue, que vers celles de la découverte : dans le premier cas, il s'agissait d'un corpus des ressources d'une culture donnée et de son catalogage; dans le second, d'un corpus heuristique ouvrant aux résultats scientifiques. Il m'apparut, cependant, que dans tous les cas s'imposait une attention minutieuse à toutes les traces, écrites ou non-écrites, laissées par une culture et que dominait à l'évidence le problème quasi-obsessionnel de les nommer : car en histoire de l'art, mon point de départ, une peinture ou une enluminure de manuscrit, une sculpture ou un édifice, chacun des exemples retenus pour servir de document, était moins le produit d'un regard que le résultat de l'acte même de nommer - d'un effort à partir de mots mis en texte. D'après l'une des principales conclusions tirées par Michael Baxandall dès son livre sur Giotto and the Orators, en 1971, ce que nous pouvons nommer nous est plus directement « visible » que ce que nous ne pouvons pas définir par des mots et seulement voir, par exemple. Dans les termes du discours sur l'objet et sur la chose, les mots rendent absent ce qu'ils devaient préserver, pourtant à tout prix, la visualité immédiate de l'œuvre d'art : le discours critique sur l'art figure, en termes de déconstruction, non pas la chose, mais l'absence de la chose, tout en étant impliqué dans sa perte. Bien qu'il se soit tenu à l'écart de la plupart des tournants 
théoriques, y compris du tournant linguistique, Baxandall n'est pas si éloigné qu'il y paraît de certaines des affirmations de Jacques Derrida (1930-2004), formulées dans certains de ses livres, entre autres La grammatologie, puis La vérité en peinture ${ }^{17}$ : si par les pouvoirs d'un texte, mis en ordre par les mots ou par l'ensemble des traces encore disponibles, on souligne la perte et son sentiment, on ne perd pas tout espoir de récupérer la chose et le sentiment de son être à travers précisément tous les signes d'un système culturel enfin recomposé ${ }^{18}$; on réaffirme le concept de l'attention de manière à pouvoir embrasser la chose, morte ou perdue, dans son reflet et, d'une certaine façon, la retrouver.

7 Il s'ensuivit un deuxième principe, en étroite relation avec le précédent, celui de la composition, dont j'aperçus vite les propriétés techniques par rapport à l'étude des objets-choses: pour les enquêtes à mener, un plan conçu sur le modèle d'un développement organique dans le temps, mais aussi dans l'espace, ce qui nécessitait d'adjoindre au corpus un projet d'atlas ${ }^{19}$; la cohérence des parties au tout, et inversement; la pertinence de chacun des membres et celle de l'ensemble qu'ils configurent, par rapport à la trame narrative, par rapport aussi à l'histoire. C'était aussi l'ordre que choisissait Leone Battista Alberti (1404-1472), dans le livre II du De Pictura, en 1435, pour travailler sur la hiérarchie des genres et revenir sur les notions établies dans l'art de la rhétorique ${ }^{20}$. Le principe de la composition articule, en effet, le passage vers le social et, plus largement, vers un moment culturel unitaire : dans le discours des humanistes sur l'art giottesque, par exemple, mais aussi chez Alberti et, ensuite, juste retour des choses et des mots, dans l'œuvre de Baxandall lui-même ${ }^{21}$. L'approche de l'unité dans la diversité même du vivant, qui devait s'avérer si féconde pour une réflexion générale, se retrouve au principe de la distinction des "périodes", et plus exactement des "périodes de regard» dans l'histoire de l'art, ce qui revient souvent sous la plume de Baxandall comme l'expression anglaise de " period eye ». L'une d'entre elles, dense et riche en développements culturels et humains, marqua tout le $\mathrm{xv}^{\mathrm{e}}$ siècle italien: tous les domaines culturels réagirent les uns par rapport aux autres pour "produire» la sensibilité dans laquelle l'art $\mathrm{du} \mathrm{xv}^{\mathrm{e}}$ siècle s'est formé, puis s'est généralisé. Différents artistes ont su utiliser les facettes de cette sensibilité, empreinte du moralisme de la prédication religieuse, de la publicité de l'art de la danse, des compétences géométriques et arithmétiques de l'art de jauger dans les transactions commerciales en cours, et de la noblesse de l'art oratoire, pour aller au-devant des attentes de leurs publics et en tenir un compte précis, au sens arithmétique du mot ${ }^{22}$ : comprendre un tableau peint, c'est s'imprégner de tous ces facteurs et restituer un document d'activité textuelle/visuelle totale, de la manière dont on peut apprendre à lire un "texte" produit dans un autre moment culturel que le sien. Chacun de ces moments particuliers délimite, ainsi qu'on l'a déjà noté, « les éléments intégrés d'une culture visuelle ${ }^{23}$ ", ce qui pour Baxandall se traduit par la force du motif,qui est l'indice d'une intentionnalité partagée, un «modèle viable » d'existence pour la chose ou pour l'objet d'art, dans les regards des artistes et de leurs clients : le motif, pattern, s'affirme dans l'échange assuré pleinement par les uns et par les autres, comme ce qui situe et exemplarise la valeur de la chose échangée, mais aussi comme ce qui implique l'identité sociale de chacun et son humanité en général. Alors qu'il théorise la compréhension sociale de la chose créée et, par extension, celle de l'art, Baxandall revient sur le concept de l'attention et l'examine sous l'angle du motif, du pattern, qui condense selon lui toute la viabilité de la transaction intervenue entre l'artiste et ses clients puis, parfois à des siècles de distance, entre l'artiste et nous ${ }^{24}$. Car chaque unité 
culturelle donnée, chaque période de regard "contextualisé », est aussi une période d'« intelligence » socialisée par la médiation de la peinture, de la sculpture, de la danse et de la rhétorique, mais aussi par toutes les activités humaines ${ }^{25}$. Au corpus et à l'atlas, posés comme les outils indispensables à la recherche, il fallait adjoindre tout de suite la prise en compte de l'économie, au sens grec du terme, mais pas seulement, puisqu'il s'agissait de regrouper toutes les approches touchant à l'humain en s'intéressant à la plus petite unité culturelle observable, au motif, capable de dévoiler l'intentionnalité de l'ensemble ${ }^{26}$. Et comme ces activités avaient des impacts mesurables sur l'environnement des sociétés, dans l'histoire et hors de l'histoire, je voulus engager un autre axe de recherches dans cette direction. Enfin, "normées " par des éléments de nature et de culture, ces sociétés avaient généré des règles et des pratiques pour servir de repères actifs à leur conduite et, plus généralement, à leur "vivre ensemble » : l'axe sur les normes du vivant, entre nature et culture, était alors inscrit dans le projet de travail futur de l'UMR.

Je m'orientais, ensuite, vers un troisième principe d'analyse qui me permettait d'aller plus avant dans la compréhension du modèle de raisonnement utilisé dans tous les domaines scientifiques, et donc au sein des différents axes de travail que je venais de distinguer, aussi bien dans les sciences humaines et sociales que dans les sciences de la terre et de la vie. En prenant pour point d'observation la connexion étroite que quelques chercheurs avaient établie avec l'archive, puis avec la mise en texte de tous les registres de l'information, je pensais qu'il fallait approfondir le champ du raisonnement analogique pour mieux en apercevoir les modes de fonctionnement et situer, au mieux, un nouveau paradigme de réflexion pour tout le groupe. Une première question se posa d'elle-même, et dès l'abord : comment comprendre la diversité de la recherche affichée par le laboratoire, dans l'unité certes, si l'on s'en tenait à la seule matrice disciplinaire ${ }^{27}$. La plupart des études qui y étaient menées reposaient sur le postulat que tout le processus de l'idéation s'inscrivait dans l'objectivité culturelle fabriquée, rejoignant en cela l'épistémologie pratique de Nelson Goodman (1906-1998), d'Edmund Leach (1910-1989), de Victor W. Turner (1920-1983), de Thomas S. Kuhn, ou encore de la philosophie de Michel Foucault (1926-1984), et tous les développements qui s'ensuivirent ${ }^{28}$. L'une des implications directes fut de concevoir la pensée comme le résultat d'échanges, voire de transactions, ce que Clifford Geertz appela une «[...] affaire de trafic dans les formes symboliques accessibles dans l'une ou l'autre communauté (langage, art, mythe, théorie, rituel, technologie, droit, et ce conglomérat de maximes, de recettes, de préjugés et d'histoires plausibles que les gens satisfaits appellent le sens commun), l'analyse de telles formes et de telles communautés est essentielle à son interprétation, non subordonnée. [...] ${ }^{29}$. " Mais une deuxième question vint à se poser très vite : était-ce un changement de paradigme au sein du groupe ou, au-delà, un changement culturel, c'est-à-dire la manière dont nous avions pensé la pensée, et dont il fallait savoir tirer toutes les conséquences, dans les résultats obtenus par le laboratoire, bien sûr, mais aussi à l'intérieur du milieu scientifique qu'il formait, pour mieux le resituer. Dans le groupe de recherche, l'ensemble des résultats restait continu, mais différemment voulu et diversement construit, parce qu'obtenu depuis des visées qui ne se recoupaient jamais, ou très peu et exceptionnellement. Face à une situation rendue aussi fluide, parfois multiple, le plus souvent dépourvue de centre, et au mieux dans un désordre ordonné, je me suis posé la question de la pertinence des explications avancées, voire celle des interprétations proposées, et je me suis interrogé sur la structure analogique, au fond, des raisonnements conduits, ce qui ne manqua pas 
de faire problème. Je me suis, en effet, aperçu du recours constant, par les uns comme par les autres - y compris par ceux qui s'en disaient le plus éloignés - aux humanités et à leurs discours, pour utiliser des analogies transférées telles quelles, avec de soi-disant propriétés explicatives, dans les sciences sociales, voire les sciences exactes. Ceci provoquait la déstabilisation des disciplines - quoi qu'on en pensât - et une croissance de la volonté de faire du sens, de produire des explications, dans tous les domaines, à partir de tous les documents, et sur tous les supports. Du reste, le constat n'avait rien d'exceptionnel et il pouvait être appliqué à bien d'autres communautés scientifiques ; mais il paraissait singulier dès lors qu'il caractérisait un groupe de chercheurs dans lequel l'archéologie et l'histoire s'affirmaient en disciplines dominantes. Le modèle heuristique des jeux et des effets de langage - et l'analogie avec le jeu doit être soulignée - engageait le groupe dans une pluralité de conduites et de conventions acceptées, et qu'il avait fini par s'approprier. Au cœur des pratiques de la recherche, et dans l'élaboration d'un discours à portée d'interprétation, régnait le régime de l'analogie ${ }^{30}$. Il m'était devenu nécessaire de le repérer pour le définir et le réintégrer dans une dynamique nouvelle de la recherche, c'est-à-dire: savoir utiliser la somme énorme de mélanges des genres disciplinaires, qui tenait lieu de transdisciplinarité ${ }^{31}$; faire valoir au contraire les "genres flous" pour re-figurer la pensée sociale du laboratoire ${ }^{32}$; le détourner du seul idéal d'explication de cas et de sens, pour le mener vers un idéal d'explication des lois et des causes, en direction d'une large réflexion sur le statut de la preuve dans les sciences de l'homme et de la société.

Or, pour moi, conserver et utiliser les "genres flous " revenait à créer les axes de recherches: les cinq axes, que j'ai déjà cités, auxquels je crus bon d'adjoindre un sixième en épistémologie, au couronnement de l'édifice projeté, pour nourrir les autres directions de travail, mais s'en nourrir tout autant, en des échanges répétés, des «transactions ${ }^{33}$.

\section{Le texte et son architecture sociale}

C'est le dernier principe de l'analyse mise en œuvre pour re-figurer la pensée sociale du laboratoire; peut-être aussi s'agit-il de la voie la plus importante pour parvenir à une dynamique qui puisse intégrer tous les axes de la recherche en les rendant bien visibles. J'essayais donc de saisir le passage du texte à son analogue, de son écriture et de sa composition en tant que discours à son action comme discours, et je m'arrêtais au concept développé par Paul Ricoeur (1913-2005) de « l'inscription », c'est-à-dire de « la fixation du sens »- demeure pour un certain temps ce qui a été dit et non pas, seul, le fait de l'avoir dit. Dans trois de ses ouvrages, plus particulièrement, Paul Ricoeur a inscrit le concept au centre de la vie des événements humains. S'étant préoccupé de découvrir une pertinence nouvelle au sens dans le discours « inscrit » sur le sol, sur du parchemin puis sur du papier, dans la pierre ou dans le bois, il en vint à explorer toute la richesse d'innovation sémantique enfermée dans la métaphore et il répondait ainsi, en 1975, au problème qu'il s'était posé, en avançant une attribution impertinente de sens, impertinente parce qu'elle dépassait proprement tout contenu fixé ${ }^{34}$. Dans sa vaste enquête sur Temps et récit, ensuite, il s'intéressait à l'invention de l'inscription et à sa mise en récit en suivant trois rapports, qu'il qualifiait de "mimétiques » : du récit au «temps agi et vécu»; du récit au «temps propre de la mise en intrigue»; enfin, du récit au «temps de la lecture ». Il éprouvait ensuite ce schéma en le confrontant avec 
l'histoire ${ }^{35}$. Enfin, reprenant le même concept, il l'évaluait au prix de la triple mise en relation à la mémoire, à l'histoire, à l'oubli ${ }^{36}$. Ceci impliquait la fixation du sens à partir du flot des événements, et non plus du cas isolé ou de l'exemple magnifié en exemplaire, et la prise en compte d'un mode de pensée plutôt familier au traducteur, à l'exégète ou à l'iconographe, voire à l'iconologue ${ }^{37}$, en réévaluant une nouvelle philologie, en quelque sorte "d'auteur » : par le besoin ressenti de réinscrire un texte avec un texte, en comprenant sous ce dernier terme toute forme d'inscription écrite ou non écrite, toute empreinte, toute trace déchiffrable ou non ${ }^{38}$. L'étude de l'inscription ne devait plus être disjointe de celle du fait d'inscrire. C'est par cette intention globale que se trouvait pleinement justifié l'axe corpus dans sa visée double, à la fois heuristique et herméneutique, devant supporter le projet dans sa plus large extension : il s'agissait, non pas de continuer la collection dérisoire d'objets juxtaposés, mais de réunir en un rassemblement organique toutes les traces disponibles pour l'ensemble de l'unité. Le nouveau philologue, qui pouvait être aussi bien l'archéologue que l'historien ou que l'historien de l'art, s'emploierait sur cette base, reformulée et retravaillée à partir des concepts, à " raccorder» entre eux les tenants des diverses pratiques et expériences ${ }^{39}$. Selon cette orientation, que je voulais constructive de l'unité du groupe, les pratiques des Chartae Burgundiae Medii Aevi ${ }^{40}$ servaient de modèle de référence et, je l'espérais, de point de départ fédérateur à l'idée bien comprise de corpus.

Et tout l'édifice s'en trouvait solidement arrimé ensemble, comme devaient l'être entre elles les pierres de l'édifice bâti, les marchandises dans la cale d'un navire, les diverses parties d'un groupe auquel je voulais donner les moyens de l'affirmation de soi. Pour terminer cette réflexion, qui est autant rapport qu'essai personnel, je reviendrai sur quelques mots de Vincent Descombes, dans son analyse du " politique »:

« [...] Lorsqu'on veut donner le principe rationnel du politique, il arrive en fait ceci :

[...] des principes fondamentaux sont en effet dégagés, principes qu'il est loisible à chacun de tenir pour des règles morales de conduite, mais aucune réduction morale $\mathrm{du}$ domaine politique n'a eu lieu, et donc aucune fondation rationnelle du domaine politique sur le roc de la seule morale personnelle, car il se découvre bientôt que ces principes contenaient déjà, sous une forme il est vrai rudimentaire, l'ordre politique lui-même ${ }^{41}$.»

\section{NOTES}

1. L'UMR est sous la triple tutelle du CNRS, de l'université de Bourgogne et du ministère de la Culture et de la Communication, et développe un très actif partenariat avec l'Inrap. Je tiens à remercier les membres du séminaire « Nature/Culture » pour la richesse des débats engagés et la pertinence des questions soulevées. Je placerai en exergue de mon essai la citation tirée de la réflexion de Jean-Pierre Vernant en rappelant ses mots : «[...] L'idée de l'autre est donc, à tout moment, liée à celle du même ; avec des passages continus entre les deux. [...] si le même se ferme sur lui-même et refuse l'autre, c'est le même, c'est l'identité, qui devient monstrueusement autre. [...] »; J.-P. VERNANT [1914-2007], La traversée des frontières. Mythe et politique, II, Paris, 2004, en part. 
«Penser la différence », repris dans Jean-Pierre Vernant. CEuvres, 2 vol., éd. M. OLENDER, Paris, 2007, en part.t. 2, p. 2318-2324.

2. Voir D. RUSSO, L. BARAY et J.-P. GARCIA (resp.), Bilan scientifique 2006-2011, UMR 5594 ARTeHIS, Dijon, 2010; D. RUSSo, "Projet scientifique de l'unité», in J.-P. GUILlAUMET, M. CLOUZOT et R. MARTINEAU (resp.), Projet de recherche 2012-2015, UMR 5594 ARTeHIS, Dijon, 2010, p. 3-20.

3. La discussion sur les concepts fut suscitée par la parution du livre de P. Descola [1949-] (Pardelà nature et culture, Paris, 2005), qui montre, à bien des égards, le point d'arrivée de cette problématique et ses reformulations possibles. Voir à présent, P. DESCOLA, "Prologue » à «La nature et ses débordements ", in S. HOUDART et O. THIERY (coord.), Humains, non humains. Comment repeupler les sciences sociales, Paris, 2011, en part. p. 17-21.

4. De manière générale, D. CUCHE, La notion de culture dans les sciences sociales, Paris, 1996 ; suivant des lignes problématiques précises, qui ont été et demeurent les miennes, C. GEERTZ [1926-2006], Local Knowledge. Further Essays in Interpretive Anthropology, New York, 1983 ; trad. de l'anglais (GB) par D. PAULME, Paris, 1986 ; L. OBADIA et L. ADDI, Clifford Geertz. Interprétation et culture, Paris, 2010. Une riche enquête sur l'histoire politique de la notion de culture, au XIX siècle : M. RIOT-SARAY, Le réel de l'utopie. Essai sur la politique au XIXe siècle, Paris, 1998. Renouvelant, mais sur le versant français cette fois, l'enquête menée dans L'âme romantique et le rêve. Essai sur le romantisme allemand et la poésie française (Marseille, 1937, 2 vol. ; Paris, 1939, 1 vol. ; Paris, 1991), par Albert Béguin [1901-1957] sur le versant germanique, dans les champs de l'histoire littéraire et de l'histoire politique, voir S. ZENKINE, L'expérience du relatif. Le romantisme français et l'idée de culture, Paris, 2011.

5. Se reporter aux propositions conceptuelles avancées par G. Balandier [1920-], dès le texte sur «Phénomènes sociaux totaux et dynamique sociale » (Cahiers internationaux de Sociologie, 30, 1961, p. 23-34; http://sociologies.revues.org/index2203.html ; consultation 3/07/2011), qui reprend et problématise les principales orientations contenues dans Sociologie actuelle de l'Afrique noire (Paris, 1951). Mêmes préoccupations du côté de F. Jacob [1920-], prix nobel de médecine (1965), dans La logique du vivant. Une histoire de l'hérédité (Paris, 1970), en particulier pour poser le questionnement sur les notions de "programme » et de "structure visible ", et ainsi affiner l'approche du cycle de la vie qui ne relève plus seulement de la biologie, pas non plus précisément de la biographie, mais de l'unité et de la diversité dans la vie sociale de l'esprit; F. JACOB, Le jeu des possibles, Paris, 1981.

6. Ces aires de résonances que tisse un laboratoire, par-delà la problématique induite par La vie de laboratoire. La production des faits scientifiques, soulignée par B. Latour et St. Woolgar (Princeton, 1986 ; trad. de l'anglais par M. BIEZUNSKI, Paris, 1988 ; 1996), renvoient à la notion élaborée au $\mathrm{XVII}^{\mathrm{e}}$ siècle par le physicien et chimiste irlandais, Robert Boyle [1627-1691], dans The Sceptical Chymist (Londres, 1661), pour rendre compte des "collèges invisibles», c'est-à-dire de ces « unités » qui composent autant de villages intellectuels, où des « données convergentes peuvent être réunies"; sur les passages cités, voir C. GEERTZ, Local Knowledge..., op. cit., en part. p. 196 («Comment nous pensons maintenant: vers une anthropologie de la pensée moderne», p. 183-204). Dans le cours du paragraphe, C. Geertz commente fort justement: «[...] des communautés d'individus aux liens multiples où quelque chose que vous trouvez au sujet de $\mathrm{A}$ vous dit aussi quelque chose de B, parce que, se connaissant l'un l'autre depuis trop longtemps et trop bien, ils sont des personnages dans les biographies l'un de l'autre.» Sur ces milieux scientifiques et les cercles d'intellectuels qu'ils formaient, cf. K. THOMAs, Religion and the Decline of Magic, Londres, 1971, en part. p. 270 et 346.

7. Sur ce terme de «l'analogie efficace », voir H.-V. WHITE, The Content of Form : Narrative Discourse and Historical Representation, Baltimore/Londres, 1990, en part. chap. 2, "The Question of Narrative in Contemporary Historical Theory », p. 26-56. Transposition du concept dans le champ littéraire avec les remarques de J. H. REID, Proust, Beckett, and Narrative, Cambridge (Mass.), 2003, 
en part. chap. 3, «Lying, Irony and Power : Proust's Deceptive Allegories » et chap. 5, « Melloy's Way : The Parody of Allegory ", p. 46-68 et 79-98.

8. Sur ces voies, J. BOUVERESSE [1940-], Wittgenstein : la rime et la raison. Science, éthique et esthétique, Paris, 1973 ; ID., Le mythe de l'intériorité. Expérience, signification et langage privé chez Wittgenstein, Paris, 1976, et Le pays des possibles. Wittgenstein, les mathématiques et le monde réel, Paris, 1988. J'ai aussi consulté du même auteur, Herméneutique et linguistique, suivi de Wittgenstein et la philosophie du langage, Paris, 1991. Dans ce champ d'étude, la réédition de P. НADOT, Wittgenstein et les limites du langage [recueil de trois textes de 1959 et d'un texte de 1962], Paris, 2004, m'a été très utile.

9. Voir C.-A. JONES et P. GALISON (dir.), Picturing Science, Producing Art, New York, 1998 ; pour une problématique développée dans le champ artistique contemporain, M. TAUSsIG, Defacement, Public Secrecy and the Labor of the Negative, Stanford, 1999.

10. B. LATOUR [1947-], «What is Iconoclash? Or Is there a world beyond the image-wars?», in B. LATOUR et P. WEIBEL, Iconoclash, Beyond the Image-Wars in Science, Religion and Art, Cambridge (Mass.), 2002, trad. de l'anglais par A. TINCELIN, in ID., Sur le culte moderne des dieux faitiches, suivi de Iconoclash, Paris, 2009, en part. p. 135-201.

11. Sur la première, voir I. HACKING, The Emergence of Probability, Cambridge (Mass.), 1975, et Entre science et réalité : la construction sociale de quoi ?, Paris, 2001 ; sur la seconde, se reporter à T. PINCH, «Observer la nature ou observer les instruments? », Culture technique, 14 (1985), p. 88-107, cité par B. LATOUR, «IConoclash», op. cit., p. 186 et n. 41, qui emploie, pour sa part, les mots de "cascade d'images"; article consulté en ligne sur le site de la revue [irevues.inist.fr/ culturetechnique], le 08/04/2011. Sur les deux notions réunies et leurs conséquences sur la vie des objets échappant à toute interprétation extérieure, M. TAMEN, Friends of Interpretable Objects, Cambridge (Mass.), 2001, en part. chap. 1, "Sensible Economics», chap. 2, «Iconoclasm», chap. 7, « Hostility to Interpretation », p. 8-27, 28-50 et 87-130.

12. M. BAXANDALL, «Exposer L'Intention. Les conditions préalables à l'exposition visuelle des objets à fonction culturelle ", Les cahiers du musée national d'Art moderne, 43 (1993), p. 35-43. Il reprenait les questions formulées par Patterns of Intention: On the Historical Explanation of Pictures, New Haven/Londres, 1985, en part. p. 13.

13. M. BAXANDALL, Giotto and The Orators: Humanist Observers of Painting in Italy and the Discovery of Pictorial Composition, 1350-1450, Oxford, 1971 ; ID., Painting and Experience in Fifteenth Century Italy : A Primer in the Social History of Pictorial Style, Oxford/New York, 1972 ; ID., The Limewood Sculptors of Renaissance Germany, New Haven/Londres, 1980.

14. En particulier, voir : C. GEERTZ, «Art as a Cultural System », Modern Language Notes 91 (1976), p. 1473-1499, repris dans Local Knowledge..., op. cit., chap. 5 (trad. fr. Savoir local, savoir global..., op. cit., en part. p. 129-137), et P. BOURDIEU [1930-2002] et Y. DESAULT, « Pour une sociologie de la perception ", Actes de la recherche en sciences sociales, 40 (nov. 1981), p. 3-9, tous deux sur Painting and Experience in Fifteenth Century Italy, dont un chapitre traduit de l'anglais par Yvette Desault, «L'œil du Quattrocento» paraissait dans le même numéro (p. 10-49). Je mentionne aussi, mais sur une autre voie d'approche, E. CASTELnUOvo, "L'histoire sociale de l'art », Actes de la recherche en sciences sociales, 4 (déc. 1976), p. 63-75.

15. En France, contemporain de Michael Baxandall, J.-L. Schefer [1938-] s'est intéressé à des champs d'études comparables, entre historicité des documents ordonnés en textes et présence des objets au monde, dans Scénographie d'un tableau (Paris, 1967) et Figures peintes. Essais sur la peinture (Paris, 1998) ; en dernier lieu, pour l'articulation du visuel et de l'objet au discours théologique dans un champ de recherche élargi, voir J.-L. SCHEFER, L'hostie profanée. Histoire d'une fiction théologique, Paris, 2007.

16. Je mentionnerai également parmi les travaux qui m'aidèrent à situer ma problématique, ceux de R.-C. Trexler [1932-2007], entre autres, Public Life in Renaissance Florence, (New York, 1980 ; Ithaca, 1991) et Church and Community, 1200-1600. Studies in the History of Florence and New Spain 
(Rome, 1987), en particulier pour la position du concept de «l'art comme expérience sociale partagée ».

17. Paris, respect. 1967 et 1978 ; notamment, La vérité en peinture fut traduite en anglais [USA] par G. Bennington, Chicago, 1987. Voir dans M. BAXANDALL, Giotto and the Orators..., op. cit., en part. p. 44 et 47 ; ainsi : « [...] the structure is that implied by the categories, the lexical and grammatical components of language. For what we can and do conveniently name is more available to us that what we cannot." De même, M. BAXANDAll, The Limewood Sculptors..., op. cit., chap. "The Period Eye ", p. 143-163 : toute l'appréciation des objets sculptés en bois passe par des mots qui catégorisent et permettent aux clients, mais aussi aux artistes, de voir les traits les plus caractéristiques des sculptures, du moins ceux qu'ils veulent distinguer comme les plus caractéristiques, parmi les traits formels, puis les traits individuels. Sur le discours de l'historien de l'art fondé sur le sens intime de la perte, R. STAMELMAN, Lost Beyond Telling: Representations of Death and Absence in Modern French Poetry, Ithaca/Londres, 1990, en part. p. 39, à partir de l'étude de Paul de Man [1919-1983], Maurice Blanchot [1907-2003] et Louis Marin [1931-1992].

18. Sur le changement de "point de vue » que cela induit dans l'analyse de l'objet-chose, et qui est aussi un changement d'ontologie du sujet, voir H. U. GUMBRECHT, Production of Presence. What Meaning Cannot Convey, Stanford, 2004, trad. de l'anglais [USA] par Fr. JAOUËN, Paris, 2010, à partir notamment de l'analyse de Martin Heidegger [1889-1976] sur l'« être-au-monde ", dans Être et Temps (1927), où il substituait ce concept au paradigme sujet/objet afin de renouer les relations possibles entre l'autoréférentialité humaine et les choses du monde ; voir G. HARMAN, Tool-Being: Heidegger and the Metaphysics of Objects, Chicago, 2002, trad. de l'anglais [USA] par O. DUBOUCLEZ, Paris, 2010. Dans les champs de l'histoire, de l'histoire de l'art et de l'anthropologie, voir E. MAGNANI et D. RUSSO, « Histoire de l'art et anthropologie, 3. Exégèse textuelle, exégèse visuelle. Autour du processus de la chose, res, dans le haut Moyen Âge ", Bucema, 13 (2009), p. 103-121 [http://cem.revues.org/index11035.html].

19. Dans Giotto and the Orators, Michael Baxandall distingue quatre niveaux dans la hiérarchisation du discours des premiers humanistes sur les choses de l'art : à la base, celui des mots; puis, celui des phrases; ensuite, celui des propositions; enfin, celui des phrases construites comme des séquences autonomes, ceci en reprenant le schéma d'organisation propre à Isidore de Séville [560/570-636], dans ses Etymologiae II, 18: «[...] fit autem ex coniunctione verborum comma, ex commate colon, ex colo periodos [...].»; op.cit., chap. 3, "Alberti and the Humanists : Composition », p. 121-139, en part. p. 131. À la hiérarchisation des périodes du discours humaniste s'articule la mise en ordre de l'espace géographique de production et de réception. De riches et stimulantes propositions pour l'étude de l'espace social de la littérature en Europe, dans F. MORETTI, Atlas du roman européen (1800-1900), Paris, 2000, et Graphes, cartes et arbres. Modèles abstraits pour une autre histoire de la littérature, Paris, 2008.

20. L.-B. ALBERTI, La peinture, texte latin, trad. fr., version ital. par T. GOLSENNE, B. PRÉVOST et Y. HERSANT (éd.), Paris, 2004.

21. M. BAXANDALL, Giotto and the Orators..., op. cit., en part. p. 6-7, pour le passage vers le social et vers un moment culturel donné : [...] By the second quarter of the fifteenth century there were a number of men in Italy able to produce verbal constructions which, though they were clearly distinguishable from real Cicero, still made use of most of the synctatical and many of the lexical facilities Cicero had enjoyed. At this moment the more lively talents among the humanists lost interest in strict ciceronianism as an end. [...]» Sur les implications socioculturelles de l'étude du latin, comme langue-signe, entre le $\mathrm{XVI}^{\mathrm{e}} \mathrm{et}$ le $\mathrm{XX}^{\mathrm{e}}$ siècle, et dans l'espace européen, voir Fr. WAQUET, Le latin ou l'empire d'un signe, $\mathrm{XVI}^{e}$-XX $\mathrm{X}^{e}$ siècle, Paris, 1998.

22. M. BAXANDALL, Painting an Experience..., op. cit., en part. p. 152, pour les exemples des peintres, Fra Angelico [1400-1455], Piero della Francesca [1415-1492], Botticelli [1445-1510], et leurs capacités à créer avec leurs publics réunis un moment culturel précis dans l'histoire des arts 
visuels. De même, entre la fin du $\mathrm{xv}^{\mathrm{e}}$ siècle et les débuts du siècle qui suit, les sculpteurs sur bois dans l'Allemagne méridionale forment, avec leurs clients, un noyau social et culturel unitaire ; ID., The Limewood Sculptors..., op. cit., chap.4, "The Market» et chap.6, "The Period Eye», p. 95-122 et 143-163.

23. J'emprunte cette expression à A. HENNION, « Michael Baxandall : les éléments intégrés d'une culture visuelle ", in ID., La passion musicale : une sociologie de la médiation, Paris, 1993, en part. p. 189-194.

24. M. BAXANDALL, Shadows and Enlightenment, New Haven/Londres, 1995, en part. p. 135-136, où, à travers le motif, se trouve comme fixée toute la complexité sociale des échanges qui font l'originalité d'un moment culturel donné ; ainsi : «[...] A viable model for thinking about some aspects of art and culture is precisely as a market in attention itself, an exchange of attentions valuable to the other. We and the artist collude in a socially institutionalized assignation to barter our respective attentions. [...] » Sur d'autres bases d'observation, à partir d'une réflexion historiographique des relations des choses à leur histoire, M. BAXANDALL, Patterns of Intention..., op. cit., en part. p. 109.

25. Pour un exemple de ce partage social dans l'intelligence des choses dans la peinture du $\mathrm{XVIII}^{\mathrm{e}}$ siècle vénitien et, plus largement, européen, S. ALPERS, M. BAXANDALL, Tiepolo and the Pictorial Intelligence, New Haven/Londres, 1994, en part. p. 40, à propos des réalisations de l'artiste à Treppenhaus, dans le Würzburg: "[...] the world presents a conundrum and his [Tiepolo] painting makes us conscious of having to work to make things out "pictorially" [...]. "

26. Voir P. ЈАСОВ, Pourquoi les choses ont-elles un sens ?, Paris, 1997 et ID., L'intentionnalité. Problèmes de philosophie de l'esprit, Paris, 2004. De même, j'ai étudié, G.-E.-M. ANscomBE [1919-2001], Intention, Londres, 2001, trad. de l'anglais [GB] par M. MAURICE et C. MICHON, et préfacé par V. DESCOMBES [1943-], en part. p. 8-20.

27. T.-S. KUHN [1922-1996], The Structure of Scientific Revolutions, Chicago [1962], 1970, trad. de l'anglais [USA] par L. MEYER, Paris [1983], 2008, qui s'interroge sur la formation de «la matrice disciplinaire » et la mise au point d'un nouveau " paradigme » dans la vie en société. Sur un cadre général d'interventions dans ce domaine de la philosophie des sciences et de la philosophie tout court, cf. G. ORIGGI et F. DARBELlay (dir.), Repenser l'interdisciplinarité, Genève, 2010, en part. I. HACKING, « Disciplinaire et satisfait », p. 77-84 ; P. JACOB, « Les réflexions d'un philosophe sur ses interactions avec un spécialiste des neurosciences », p. 125-138.

28. Sur ces façons de penser qui sont aussi des manières d'agir sur les problématiques définies en sciences et en sciences humaines, voir la démonstration de G. HOLTON [1922-], L'imagination scientifique, Paris, 1981.

29. C. GEERTZ, "Comment nous pensons maintenant", op. cit., en part. p. 190 ; et avec cette remarque pour conclure le paragraphe: «[...] C'est une question de concevoir connaissance, émotion, motivation, perception, imagination, souvenir... n'importe quoi, comme étant, en ellesmêmes et directement, des affaires sociales. »

30. Du point de vue de l'analyse discursive, voir les descriptions de J.-L. AUSTIN [1911-1960], How to Do Things with Words, Oxford, 1962 [éd. James O. URMSON]. Sur l'analogie comme mode de conduite interactive, et ses rapports avec le jeu, le théâtre et les formes sociales de symbolisation en général, se reporter aux travaux d'E. GOFFMAN [1922-1982], La mise en scène de la vie quotidienne, t. 1 (La présentation de soi) et t. 2 (Les relations en public), Paris, 1973.

31. Sur ces difficultés, et pour une approche théorique, Le défi de l'inter-et transdisciplinarité. Concepts, méthodes et pratiques innovantes dans l'enseignement et la recherche, Lausanne, 2008.

32. J'emprunte l'expression des " genres flous » à C. GEERTZ, «Blurred Genres: The Refiguration of Social Thought", The American Scholar, 29/2 (printemps 1980); repris dans ID., Local Knowledge..., op. cit., chap. 1, trad. de l'anglais par D. PAULME, in ID., Savoir local, savoir global..., op. cit., p. 27-47 ; en part. p. 27, cette remarque : « [...] En outre, je ne pense pas seulement que ces 
choses sont vraies, je pense qu'elles sont vraies ensemble; et que la responsabilité en incombe au changement culturel, tel est mon sujet : la refiguration de la pensée sociale. »

33. Pour l'axe d'épistémologie, tel que je l'envisageais alors, j'ai suivi N.-R. HANson, Patterns of Discovery, Cambridge, 1958, sur les bases et les fonctions des théories scientifiques, ainsi que pour l'emploi du mot anglais pattern et ses différents contextes d'utilisation; j'ai aussi lu C. G. HEMPEL, Aspects of Scientific Explanation and Other Essays in the Philosophy of Science, New York, 1965, notamment pour l'étude de la formation des concepts et le moteur de l'explication dans les sciences.

34. P. RICOEUR, La métaphore vive, Paris [1975], 1997. Sur la question des rapports au champ mimétique, H. V. WHITE, Figural Realism : Studies in the Mimesis Effect, Baltimore/Londres, 2000, en part. chap. 1, «Literary Theory and Historical Writing " et chap. 2, « Historical Emplotment and the Problem of Truth in Historical Representation », p. 1-26 et 27-42.

35. P. RICOEUR, Temps et récit, t. 1 (L'intrigue et le récit historique), Paris [1983], 1991 ; pour une discussion du concept de «l'inscription » aux prises avec l'histoire envisagée comme un système culturel, voir D. LABORDE (dir.), Désirs d'histoire: politique, mémoire, identité, Paris, 2009, en part. G. LENCLUD, «Les cultures humaines et le bateau de Thésée. Le problème de l'identité des cultures dans le temps", p. 221-248. Sur les rapports entre le discours fixé et la "représentation» historique, H. V. WHITE, The Content of the Form..., op. cit., en part. chap. 2, "The Question of Narrative in Contemporary Historical Theory " et chap. 7, "The Metaphysics of Narrativity: Time and Symbol in Ricoeur's Philosophy of History », p. 26-55 et 169-184.

36. H. V. WHITE, La Mémoire, l'Histoire, l'Oubli, Paris [2000], 2003, en part. toute la deuxième partie, «L'Histoire », qu'il situait dans une épistémologie des sciences historiques.

37. .Je renvoie aux propositions d'étude sur textuel/visuel, et inversement, de W. J. Th. MITCHELL, Iconology. Image, Text, Ideology, Chicago, 1986, trad. de l'anglais [USA] par M. BoIDY et St. ROTH, Paris, 2009.

38. C. GINZBURG [1939-], Occhiacci di legno. Nove riflessioni sulla distanza, Milan, 1998, trad. de l'italien par P.-A. FABRE, Paris, 2001 ; ID., Il filo e le tracce, Milan, 2006, trad. de l'italien par M. RUEFF, Paris, 2010 [recueil de textes parus entre 1998 et 2003] ; L. GIAVARINI, «Carlo Ginzburg dans la forêt de la tradition littéraire ", Acta Fabula, Dossier critique : "Faire et refaire l'histoire", [http://www.fabula.org/revue/document6398.php], consulté le 17/09/2011.

39. É. DURIG, Faux raccords. La coexistence des images, Arles, 2010, pour le concept de " raccord», que j'utilise : en part. la $3^{\mathrm{e}}$ partie, « Connexions », p. 153-205.

40. Les Chartae Burgundiae Medii Aevi [CBMA] furent lancées par E. Magnani et M.-J. GasseGrandjean en octobre 2004, et poursuivent leur travail sous la direction d'E. Magnani, et en coresponsabilité avec M.-J. Gasse-Grandjean.

41. V. DESCOMBES, «Philosophie du jugement politique », La Pensée politique, juin 1994 ; repris dans Philosophie du jugement politique. Débat avec Vincent Descombes, Paris, 2008, en part. p. 7-48, citation p. 47-48. En fin de citation, les italiques sont miennes.

\section{INDEX}

Mots-clés : interdisciplinarité 